\title{
Percepção dos profissionais de saúde sobre a criança com doença crônica
}

\author{
Perception of health professionals about children with chronic illness \\ Percepción de los profesionales de la salud sobre los niños con enfermedades crónicas
}

Recebido: 21/05/2021 | Revisado: 29/05/2021 | Aceito: 03/06/2021 | Publicado: 18/06/2021

\author{
Viviane Marten Milbrath \\ ORCID: https://orcid.org/0000-0001-5523-3803 \\ Universidade Federal de Pelotas, Brasil \\ E-mail: vivianemarten@ hotmail.com \\ Ruth Irmgard Bärtschi Gabatz \\ ORCID: https://orcid.org/0000-0001-6075-8516 \\ Universidade Federal de Pelotas, Brasil \\ E-mail: r.gabatz@yahoo.com.br \\ Bárbara Hirschmann \\ ORCID: https://orcid.org/0000-0002-6439-3574 \\ Universidade Federal de Pelotas, Brasil \\ E-mail: babi.h@live.com \\ Roberta Hirschmann \\ ORCID: https://orcid.org/0000-0002-3775-0310 \\ Universidade Federal de Pelotas, Brasil \\ E-mail: r.nutri@hotmail.com \\ Vera Lúcia Freitag \\ ORCID: https://orcid.org/0000-0002-5897-7012 \\ Universidade de Cruz Alta, Brasil \\ E-mail:verafreitag@hotmail.com \\ Vanessa Acosta Alves \\ ORCID: https://orcid.org/0000-0002-1209-672X \\ Universidade Federal de Pelotas, Brasil \\ E-mail: vanessaacostaalves@hotmail.com \\ Jéssica Cardoso Vaz \\ ORCID: https://orcid.org/0000-0002-2581-1091 \\ Universidade Federal de Pelotas, Brasil \\ E-mail: jessica.cardosovaz@gmail.com
}

\begin{abstract}
Resumo
Objetivo: Conhecer a percepção dos prossionais da saúde quanto ao cuidado prestado à criança com condição crônica. Metodologia: Trata-se de uma pesquisa descritivo exploratória com abordagem qualitativa, realizada com cinco profissionais de saúde referência para familiares de crianças com doença crônica. A coleta dos dados ocorreu por meio da entrevista semi-estruturada e foram interpretados por meio da análise temática. Resultados: Emergiram duas unidades temáticas: A reconhecida importância do cuidado multiprofissional contrapondo-se a realidade vivenciada; $\mathrm{e}$ A família percebida como o principal facilitador ou dificultador do cuidado à criança com doença crônica. Discussão: A criança com doença crônica necessita ser assistida por uma equipe multidisciplinar, em um acompanhamento contínuo nos serviços de saúde, contudo, isso foi identificado como uma lacuna neste estudo. Percebeu-se também a representatividade da família no cuidado, através de uma rede social fortalecida e bem definida, atendendo a essa criança sob a ótica biopsicosocioespiritual. Considerações finais: $\mathrm{O}$ acesso e a disponibilidade de profissionais preparados são fundamentais para atender às demandas de crianças com condições crônicas, sendo imprescindível um cuidado ampliado, contínuo e resolutivo, capaz de identificar as necessidades biológicas, emocionais e sociais dessa população, efetivando o acolhimento integral.
\end{abstract}

Palavras-chave: Criança; Doença crônica; Pessoal de saúde; Família; Saúde da criança.

\begin{abstract}
Objective: To know the perception of health professionals regarding the care provided to children with chronic conditions. Methodology: This is a descriptive exploratory research with a qualitative approach, carried out with five health professionals who are referrals for family members of children with chronic disease. Data collection took place through semi-structured interviews and were interpreted through thematic analysis. Results: Two thematic units emerged: The recognized importance of multiprofessional care, in contrast to the reality experienced; and The family perceived as the main facilitator or hinderer in the care of children with chronic illness. Discussion: The child with chronic illness needs to be assisted by a multidisciplinary team, in a continuous monitoring in the health services, however, this was identified as a gap in this study. It was also noticed the representativeness of the family in care, through a strengthened and well-defined social network, serving this child from the biopsychosocio-spiritual
\end{abstract}


perspective. Final considerations: Access and availability of trained professionals are essential to meet the demands of children with chronic conditions, and expanded, continuous and resolving care is essential, capable of identifying the biological, emotional and social needs of this population, making full care possible.

Keywords: Child; Chronic disease; Health personnel; Family; Child health.

\section{Resumen}

Objetivo: Conocer la percepción de los profesionales de la salud sobre la atención brindada a los niños con enfermedades crónicas. Metodología: Se trata de una investigación exploratoria descriptiva con enfoque cualitativo, realizada con cinco profesionales de la salud que son referencia para los familiares de niños con enfermedad crónica. La recolección de datos se realizó a través de entrevistas semiestructuradas y se interpretaron mediante análisis temático. Resultados: Surgieron dos unidades temáticas: La reconocida importancia del cuidado multiprofesional, en contraste con la realidad vivida; y La familia percibida como el principal facilitador u obstaculizador en el cuidado de los niños con enfermedades crónicas. Discusión: El niño con enfermedad crónica necesita ser asistido por un equipo multidisciplinario, en un seguimiento continuo en los servicios de salud, sin embargo, esto fue identificado como un vacío en este estudio. También se notó la representatividad de la familia en cuidado, a través de una red social fortalecida y bien definida, atendiendo a este niño desde la perspectiva biopsicosocio-espiritual. Consideraciones finales: El acceso y disponibilidad de profesionales capacitados es fundamental para atender las demandas de los niños con enfermedades crónicas, y es fundamental la atención ampliada, continua y resolutiva, capaz de identificar las necesidades biológicas, emocionales y sociales de esta población, posibilitando una atención integral.

Palabras clave: Niño; Enfermedad crónica; Personal de salud; Familia; Salud del niño.

\section{Introdução}

A vivência do adoecimento e da confirmação do diagnóstico de uma patologia durante a infância é um momento delicado, que se reveste de nuances de maiores proporções quando esse diagnóstico se associa à confirmação de uma doença crônica (Milbrath, Motta, Gabatz \& Freitag, 2017). A vida das crianças que passam a conviver com a cronicidade são marcadas por inúmeras transformações, as quais podem conferir limitações e exigir mudanças de hábitos e atitudes em função do tratamento e dos cuidados inerentes a sua condição de saúde (Souza et al, 2021).

A existência de uma doença crônica impõe a necessidade de um cuidado contínuo e efetivo para que se evite a piora do quadro clínico (Silva, Reichert, Souza, Pimenta \& Collet, 2018). Dessa forma, é fundamental que as crianças e suas famílias possam contar com o apoio dos profissionais da saúde, para darem conta das demandas oriundas da condição crônica (Machado et al, 2018). A doença crônica na infância causa uma série de mudanças na rotina familiar e a necessidade de (re)adaptações (Dias et al, 2020), entre elas destacam-se as financeiras, decorrentes dos tratamentos, e também porque em alguns casos um dos membros da família (na maioria das vezes a mãe), deixa o emprego para cuidar da criança, além de adaptações na vida social, pois por vezes não há como manter a mesma rotina que existia anteriormente (Milbrath, Motta, Gabatz \& Freitag, 2017; Dias et al, 2018).

As adversidades provocadas pela condição crônica na infância e as vulnerabilidades vividas por essa população são questões relevantes para a reflexão e a organização dos serviços de saúde. A escuta qualificada, o acolhimento e a ética são fatores importantes no cuidado de pessoas com condições crônicas, assim como o atendimento compartilhado entre os profissionais e os serviços da rede de atenção à saúde, para que seja possível repensar o cuidado à criança com doença crônica que vem sendo ofertado, visando sua melhoria (Alves, Amendoeira \& Charepe, 2017).

Para diminuir a sobrecarga vivenciada pela família ao cuidar das necessidades oriundas da condição crônica da criança, é fundamental que esta possa contar com um profissional de referência e/ou serviço de saúde de apoio, não apenas em uma perspectiva biomédica, de prescrever e seguir uma prescrição, mas de uma relação de cuidado em que o profissional e a família possam compartilhar informações, tomar decisões conjuntas, em que o êxito técnico tenha como um de seus fundamentos os sucessos práticos almejados. 
Considerando esses pressupostos, estabeleceu-se a seguinte questão de pesquisa: Como os profissionais da saúde percebem o cuidado à criança com condição crônica? Com o objetivo de conhecer a percepção dos profissionais da saúde quanto ao cuidado prestado à criança com condição crônica.

\section{Metodologia}

Trata-se de uma pesquisa descritivo exploratória com abordagem qualitativa (Minayo, 2013). Essa constitui um recorte de uma pesquisa multicêntrica desenvolvida em quatro municípios do Rio Grande do Sul (Porto Alegre, Santa Maria, Palmeira das Missões e Pelotas) e um município de Santa Catarina (Chapecó), intitulada Vulnerabilidades da criança e adolescente com doença crônica: cuidado em rede de atenção à saúde, sendo que o recorte aqui apresentado se refere ao município de Pelotas (RS). A coleta das informações ocorreu no ano de 2019, com profissionais de saúde referência para familiares/cuidadores de crianças com doença crônica. Os critérios de inclusão foram: ser profissional de saúde que presta cuidado a crianças (6-12 anos) diagnosticadas com doença crônica; ser indicado como referência no cuidado pelos familiares/cuidadores das crianças com doença crônica.

Os profissionais de saúde foram contatados e convidados para participar da pesquisa após explicação dos objetivos e do desenvolvimento da mesma. A entrevista foi agendada em local privativo no ambiente de trabalho dos profissionais e iniciada após leitura e assinatura do Termo de Consentimento Livre e Esclarecido.

Das quinze famílias acessadas pela pesquisa e incluídas no estudo, oito indicaram um profissional de referência no cuidado da criança com doença crônica, sendo que cinco, dos oito profissionais de saúde indicados, aceitaram participar, dos quais: uma enfermeira, uma técnica de enfermagem, uma médica, uma nutricionista e uma psicóloga.

Os preceitos éticos preconizados na Resolução 466/12 do Conselho Nacional de Saúde do Ministério da Saúde, que discorre sobre aspectos da pesquisa envolvendo seres humanos, foram respeitados (Brasil, 2013). Antes da realização da pesquisa o projeto foi encaminhado para a Comissão de Pesquisa da Escola de Enfermagem da Universidade Federal do Rio Grande do Sul/COMPESQ e Comissão de Ética em Pesquisa da Universidade Federal do Rio Grande do Sul, sendo aprovado pelo CAEE 54517016.6.1001.5327, sob o parecer 1.523.198.

A coleta das informações foi realizada por meio de entrevista semi-estruturada, com questões com questões fechadas traçando o perfil dos entrevistados e questões abertas: Fale-me sobre as facilidades e dificuldades que você encontra no que se refere ao cuidado da saúde da criança com doença crônica na unidade de saúde; fale sobre as redes de apoio que os profissionais necessitam para acompanhar a criança com condição crônica. As entrevistas foram gravadas com utilização de aparelho de celular e posteriormente transcritas na íntegra, tendo duração média de aproximadamente 25 minutos.

Para a interpretação dos resultados foi utilizada a análise temática, essa se constitui de seis fases: $1^{\circ}$ fase: familiarização com os dados, $2^{\circ}$ fase: geração dos códigos iniciais, $3^{\circ}$ fase: busca por temas, $4^{\circ}$ fase: revisão dos temas, $5^{\circ}$ fase: definição e nomeação dos temas, $6^{\circ}$ fase: produção do relatório (Clarke \& Braun, 2013).

Após a análise dos dados foram elencados dois temas principais para apresentação dos resultados: A reconhecida importância do cuidado multiprofissional contrapondo-se a realidade vivenciada; A família percebida como o principal facilitador ou dificultador do cuidado à criança com doença crônica.

\section{Resultados}

Os profissionais de saúde incluídos neste estudo foram indicados pelas famílias das crianças com doenças crônicas, geralmente pela mãe, no entanto, cabe destacar que das quinze famílias acessadas pela pesquisa e incluídas no estudo apenas oito cuidadores indicaram um profissional de referência no cuidado da criança com doença crônica. Entre esses, houve uma recusa e três perdas pela impossibilidade de contato com esses profissionais após diversas tentativas, em dias e horários 
diferentes, porém, destaca-se que a maioria das famílias (seis) não indicou um profissional de referência, porque a criança não recebe acompanhamento ou é atendida apenas pelo plantonista quando o quadro de saúde agrava e é necessário recorrer ao serviço de saúde. Esse dado evidencia uma situação preocupante, uma vez que as condições crônicas de saúde apresentam episódios de agudização e o cuidado contínuo realizado por um profissional de referência deve ser valorizado, podendo minimizar as intercorrências.

Portanto, participaram do estudo cinco profissionais da saúde: uma enfermeira, uma médica, uma técnica de enfermagem, uma nutricionista e uma psicóloga, todas do sexo feminino, com idades que variaram de 30 a 50 anos, três eram casadas e duas solteiras, o tempo de atuação na unidade variou de 2 anos e 6 meses à 19 anos.

\section{A reconhecida importância do cuidado multiprofissional contrapondo-se a realidade vivenciada}

Ao dialogar com os participantes foi destacado por eles a importância da equipe de saúde no acompanhamento da criança com doença crônica. Como se pode ver no depoimento que segue:

Na verdade, toda criança crônica, ela demanda uma equipe multidisciplinar. Acho que isso é algo muito importante e que é uns dos diferenciais aqui da FAMED (ambulatório da faculdade de medicina) porque os pacientes têm aqui. (PS3)

Em contrapartida, outra profissional da saúde indicada pela família como referência, mas que atua na escola, evidencia a falta de outros profissionais no manejo e no cuidado da criança com doença crônica.

A princípio nós ficamos bastante angustiadas e preocupadas, porque é o nosso primeiro caso de Diabetes e a nossa preocupação era saber quando estava alta e quando que estava baixa, quando era necessário aplicar insulina, quando ele tinha que comer o docinho para regularizar, então essa foi a nossa principal angústia. [...] ano passado como ainda era início, dosagem, quanto que ele tinha que aplicar de insulina, então ainda tinha muito descontrole. Então o (nome da criança) muitas vezes se sentiu mal aqui na escola. Então ele vinha seguido para fazer o testezinho (HGT) aqui conosco, quando ele se sentia mal ele já sentia os sintomas, então ele nos procurava. Teve momentos que a gente teve que recorrer à família para vir buscar [...]. Então assim, o início foi mais difícil, porque realmente era o novo, nós não conhecíamos. (PS5)

Os profissionais de saúde explicitaram ainda que enfrentam dificuldades no cuidado da criança com doenças crônicas pelo fato da alta demanda e do quadro profissional reduzido.

Dificuldade é que em alguns momentos são poucos profissionais, os horários que se tem são poucos, e porque às vezes o poder público acha que tudo tem que ficar a cargo da universidade, então não tem profissionais em outros locais que façam isso, e aí acaba que a demanda da universidade está muito, muito, muito suprimida por causa disso [...]. Mas acho que os pacientes gostam muito de serem atendidos aqui, eles se sentem muito bem. E a gente sempre tenta fazer o máximo, o possível e o impossível. (PS3)

\section{A família percebida como o principal facilitador ou dificultador do cuidado à criança com doença crônica}

Com a análise dos dados foi possível identificar as dificuldades e as facilidades que os profissionais da saúde identificam para realizar o acompanhamento e o cuidado à criança com condição crônica. Os participantes destacaram, em geral, que as facilidades no cuidado se davam em relação à assiduidade, à adesão ao tratamento, ao interesse e à atenção dos familiares, com destaque para a figura materna, como se pode ver nos depoimentos a seguir: 
Ele é uma criança super tranquila [...] a família é também bem atenciosa, bem interessada. (PS2)

Facilidade acho que é a disponibilidade da mãe. (PS4)

Ter apoio da família. (PS5)

Ademais, os relatos também evidenciam que a presença do pai seria fundamental para o enfrentamento da doença, através do apoio e do cuidado, bem como de seu comparecimento às consultas de rotina.

A dificuldade é o pai, o pai não comparecer às consultas, nunca compareceu e ele é, [...] uma criança diferente. [...] é a mãe sozinha. Por isso que eu acho também que o [nome da criança] sente [...]. Eu acho que se o pai apoiasse mais, seria mais fácil também eu acho. [...] claro, que agora eu disse para ela, que o psicólogo fazendo o acompanhando, vai chamar o pai, vai conversar com o pai, aí o pai vai abraçar a causa junto né? Vai dar o apoio que ele precisa [...] que a mãe precisa e o [nome da criança]. Porque a mãe sente a falta do pai, se a mãe tiver o apoio do pai ela se fortalece também para ajudar o [nome da criança]. (PS4)

A ausência da rede social secundária, representada por outros profissionais ou instituições de saúde também foi destacada como uma dificuldade.

Tem muito a função psicológica que o [nome da criança] também estava esperando a psicóloga e estava fazendo muita falta e aí conversando muito com a mãe a gente chegou à conclusão que faltava até apoio para ela, porque ela estava com dificuldade de lidar com nome [nome da criança] então eu acho que a parte psicológica [...] o psicólogo, está faltando muito esse profissional [...]. Porque a dificuldade dele é controlar também eu acho, controlar o fator emocional. (PS4)

Um dos participantes também ressaltou a dificuldade vivida pelas crianças ao experienciarem o adoecimento e a condição crônica na infância:

É, na infância [pausa na fala] para elas isso acaba se tornando um problema. Que aí tu faltas a aula, não vê o coleguinha daquele dia, perde a matéria, tem que recuperar a aula. Sem contar que eles vêm para cá para serem picados. Ai eles não gostam. (PS1)

\section{Discussão}

A alteração no perfil de morbidade na infância, com aumento das condições crônicas de saúde, requer uma reorganização no atendimento dessas crianças, voltando o olhar as suas necessidades, em busca de um cuidado contínuo que proporcione qualidade de vida (Duarte et al, 2015).

A criança com condição crônica necessita de um acompanhamento contínuo nos serviços de saúde, para que se evite o agravamento do seu quadro. O fato das crianças não serem acompanhadas por um serviço de saúde ou profissional faz com que vivenciem uma situação de vulnerabilidade social, pois tem seu acesso aos serviços de saúde limitados (Vaz, 2018).

Os participantes salientam que a criança com doença crônica necessita ser acompanhada por uma equipe multidisciplinar, um dos profissionais relata que o local em que trabalha fornece o atendimento especializado às crianças crônicas. No entanto, cabe destacar que esta não é a realidade do atendimento recebido por todas as crianças, como foi 
observado neste estudo, pois muitas vezes elas não têm um profissional e/ou serviço de referência, que conheça seu histórico clínico, sendo atendidas por profissionais diferentes ou plantonistas, ou seja, não recebem acompanhamento continuado.

Outra pesquisa também destacou a importância do acompanhamento de uma equipe multiprofissional para crianças e adolescentes com necessidades especiais de saúde, sendo que, muitas vezes, as famílias vivenciam uma verdadeira via sacra para conseguirem ser acompanhados por um serviço especializado de saúde (Milbrath, Motta, Gabatz \& Freitag, 2017).

Diante do aumento significativo dos casos de crianças com doenças crônicas é indispensável salientar a importância do cuidado continuado, bem como da qualificação dos serviços de atenção primária à saúde e de seus profissionais, no sentido de promover competências na assistência que possam suprir as demandas singulares que surgem na condição crônica.

Sendo assim, é fundamental que os profissionais de saúde estejam preparados para assistir às demandas decorrentes da doença crônica, para que consigam acolher a criança e suas famílias em todo o processo, desde os momentos iniciais com cuidado contínuo, proativo e resolutivo (Nóbrega, Damasceno, Rodrigues, Reichert \& Collet, 2013; Nóbrega et al, 2017), uma vez que a presença de doenças crônicas, o acesso dificultado aos serviços e a ausência de um profissional de saúde como referência repercute negativamente na saúde da criança, na sua qualidade de vida e no prognóstico.

Entretanto, nem sempre os serviços de saúde, como a atenção primária à saúde, são procurados pelas famílias em situações de doença, o que impossibilita a construção de vínculo com esse serviço, uma vez que a construção do vínculo se dá através da busca pelos familiares das crianças com doença crônica por resolutividade no cuidado na rede de atenção à saúde (Silva, Reichert, Souza, Pimenta \& Collet, 2018). Assim, a falta de contato com as famílias das crianças com doença crônica dificulta o acompanhamento e o cuidado dos profissionais (Souza, Nóbrega \& Collet, 2020).

A criança com doença crônica e sua família têm demandas de cuidado singulares em cada fase da doença, que merecem a atenção dos profissionais de saúde. Para que esse cuidado seja efetivo torna-se necessária a sensibilidade no acolhimento através escuta qualificada das necessidades da família, cuidado apoiado, ampliado e contínuo e orientação, que a fortaleçam para enfrentar a doença do filho (Nóbrega, 2017; Machado et al, 2015).

A relação entre profissional de saúde e paciente ou com um serviço de saúde de referência, que promova resolutividade de suas necessidades, pode contribuir para melhores resultados em termos de diagnóstico, tratamento e gerenciamento das ações de saúde, produzindo um efeito positivo na situação de saúde da criança ou do adolescente com doença crônica.

Complementarmente, a relação de profissionais de saúde com familiares e crianças com doença crônica é muito relevante, pois os discursos e práticas desses profissionais geram grande impacto na vida destas famílias e de seus filhos (Pais $\&$ Menezes, 2019).

Os participantes destacaram a importância de ter a família como parceira no cuidado à criança. Inúmeros trabalhos também salientam a relevância da família para o cuidado à criança e ao adolescente com necessidades especiais de saúde (Milbrath, Motta, Gabatz \& Freitag, 2017; Dias et al, 2020; Souza, Nóbrega \& Collet, 2020). O vínculo existente entre profissional da saúde e família é identificado como apoio social para o enfrentamento de dificuldades, compreendendo o diálogo e a escuta como duas das ferramentas utilizadas no acolhimento da família para o estabelecimento e promoção do vínculo social entre equipe e família (Machado et al, 2018).

Nessa perspectiva, entende-se o diálogo como uma maneira de vincular família e equipe, além de ser considerada uma forma de cuidado à família, tornando-a valorizada ao compartilhar experiências, fazendo com que se sinta parte do processo de cuidado (Machado et al, 2018). Vale também ressaltar o destaque da figura materna como responsável por facilitar esse processo de assistência à criança e ao adolescente com condição crônica, o que vai ao encontro de outras pesquisas que encontraram esse papel atribuído a mãe (Dantas, Neves, Ribeiro, Brito \& Batista, 2019; Freitag, Milbrath \& Motta, 2020). 
Outro estudo que objetivou descrever as necessidades de apoio dos pais em relação aos problemas que vivenciam ao ter um filho com doença crônica, evidenciou que eles necessitam de informações precisas sobre a doença e o tratamento, para que possam (re)organizar sua vida (Geense, Van Gall, Knoll, Cornelissen \& Van Achterberg, 2017).

Desse modo, destaca-se a importância da rede social e de apoio no cuidado da criança com doença crônica e sua família. Entende-se por rede social um conjunto de relações interpessoais, a dimensão estrutural ou institucional ligada a um indivíduo e a partir dessa rede as pessoas estabelecem vínculos de amizade e informações, recebem ajuda emocional, apoio material e de serviços os quais contribuem de forma positiva na manutenção da saúde. Quanto a sua natureza, as redes sociais podem ser: primária, caracterizada pelas relações familiares, de amizade ou vizinhança, ou secundária, representada principalmente pelas pessoas vinculadas às instituições de saúde, educação ou de assistência social (Barbosa, Reis, Lomba, Alves \& Braga, 2016; Silva, Moura, Albuquerque, Reichert \& Collet, 2017).

Além disso, estar vulnerável para uma determinada doença depende das condições biológicas e individuais de cada pessoa, bem como da sua interação com o meio ao qual pertence e com a sociedade, oferecendo ou não, respostas a suas necessidades (Benedetto \& Silveira, 2013). Dessa forma, estar vulnerável ou não, está fortemente relacionado com as condições familiares para seus cuidados, de forma que ter suporte de um cuidador pode auxiliar a reduzir a situação de vulnerabilidade.

Todavia, o cuidado dispensado às crianças e aos adolescentes com doenças crônicas é um processo desafiador, pois tem fatores importantes envolvidos no contexto, como: a gravidade da doença, a maneira como os familiares administram a situação e a forma de acesso aos cuidados na rede de atenção à saúde. Ainda, considera-se o contexto social e econômico no qual as pessoas estão inseridas e aspectos relacionados ao ambiente e à cultura (Araujo, Santos, Neves, Cardoso \& Nascimento, 2020).

Por outro lado, a rede social, neste caso a ausência de uma rede de apoio, também foi relatada pelos profissionais de saúde como uma dificuldade no cuidado da criança com doença crônica. Um dos participantes também mencionou como dificuldade a ausência de apoio paterno vivenciada pelas famílias.

A ausência da figura paterna foi destacada pelo participante, como prejudicial para a criança e também para a mãe. Segundo pesquisas a atitude do pai costuma atingir os demais membros da família, especialmente a mãe (Leite et al, 2020; Pedrosa et al, 2020).

Para realizar o cuidado à criança com condição crônica é fundamental que o cuidador tenha o apoio emocional de seu parceiro, da família, de amigos, de colegas e de profissionais de saúde para lidar com a condição do filho (Freitag, Milbrath \& Motta, 2020). O cuidador geralmente prioriza o cuidado da criança em detrimento a sua vida pessoal (Freitag, Milbrath \& Motta, 2020; Geense, Van Gall, Knoll, Cornelissen \& Van Achterberg, 2017), e por isso muitas vezes, se encontra exausto, deprimido, estressado e com baixa qualidade de vida, o que pode ter um impacto negativo nos resultados de saúde de seu filho. O apoio com o objetivo de prevenir e reduzir o estresse parental é essencial.

Portanto, é necessário ter uma visão dos problemas e necessidades de apoio desses pais (Geense, Van Gall, Knoll, Cornelissen \& Van Achterberg, 2017). Estudo abordou a respeito da participação paterna nesse contexto e percebeu que, aos poucos o que era considerado um cuidado apenas materno vem tomando outras proporções, sendo que o cuidado paterno com questões como sono, higiene, alimentação e alguns cuidados relacionado a própria doença crônica possui papel fundamental, visando a não sobrecarga materna e o estabelecimento de vínculo e apoio refletido em ambos. Entretanto, há casos em que a figura paterna é vista como despreparada para auxiliar nesse cuidado e, portanto, as mães acabam se sobrecarregando por não haver uma confiança no envolvimento paterno com a situação ou então sentem que esse é um papel unicamente delas mesmas e acabam por colocar a responsabilidade sobre si, tornando essa tarefa ainda mais complicada, visto que o fazem da mesma maneira com relação aos cuidados domésticos e às demais funções (Ramos, Nobrega, Fernandes, Machado \& Collet, 2017). 
A divisão de funções e tarefas no cuidado da criança ou do adolescente com doença crônica, no contexto familiar, é uma das estratégias utilizadas pelas famílias, a fim de suprir as demandas que estes necessitam, havendo participação ativa de pais e mães no cuidado e na assistência ao filho (Salvador et al, 2015).

É fundamental ressaltar a importância da rede familiar como um todo nesse cuidado, estudos destacam que quando existe um apoio mútuo entre o casal o processo de adaptação à condição crônica da criança e/ou adolescente fica facilitado (Ramos, Nobrega, Fernandes, Machado \& Collet, 2017; Martins, Azevedo \& Afonso, 2018).

Um participante destacou a necessidade de contar com uma equipe multiprofissional para atender todas as necessidades da criança e do adolescente como um ser humano biopsicosocioespiritual. Alguns estudos demonstram a importância da rede social no cuidado de crianças com doenças crônicas, demonstrando que uma rede fortalecida contribui para o enfrentamento da condição crônica (Freitag, Milbrath \& Motta, 2020; Cabral \& Moraes, 2015; Domenico \& Castillo, 2017). Essa rede de apoio deve lançar um olhar à família, especialmente ao cuidador principal, que em geral, é a mãe (Cardoso, dos Santos, de Araújo, Neves \& do Nascimento, 2021), que acumula várias tarefas.

A fragilidade na assistência decorrente da ausência ou número reduzido de profissionais destaca uma situação de vulnerabilidade programática vivenciada por crianças e adolescentes com condição crônica e suas famílias. A vulnerabilidade programática diz respeito ao grau e à qualidade dos recursos disponíveis para a saúde (Ayres, Calazans, Saletti \& França, 2006). É fundamental um investimento por parte do Estado no emprego de recursos capazes de melhorar a assistência prestada e o acesso aos serviços de saúde para crianças/adolescentes com condições crônicas e suas famílias.

A demanda de atendimento superior ao que seria possível de ser realizado pelo número de profissionais existentes no serviço de saúde, que são poucos, também foi relatada por profissionais de saúde e identificada em outro estudo. Nesse, o fluxo elevado de usuários sobrecarrega os profissionais e dificulta a realização de atividades, atendimentos e ações de prevenção, que se fossem realizados por maior número de profissionais poderiam beneficiar as crianças em condições crônicas (Duarte et al, 2015).

Quando há escassez de profissionais de saúde, o cuidado é praticado de forma fragmentada, centrando-se nas necessidades pontuais das crianças com necessidades especiais em saúde, ainda existe a insuficiência na formação e na qualificação profissional, que podem interferir no planejamento e na implementação do cuidado à criança (Lino et al, 2020).

O profissional de saúde de referência da Família 5 é o psicólogo, no entanto, ele não pertence a um serviço de saúde, e sim a uma escola. Esse destacou a dificuldade vivenciada pela ausência de um profissional habilitado dentro da escola para realização de procedimentos simples, como o Hemoglicoteste (HGT).

Outras pesquisas com famílias de crianças e adolescentes com necessidades especiais de saúde também reforçaram que seria importante e um facilitador do cuidado se nas escolas tivessem um profissional da saúde como o enfermeiro, para atuar na educação em saúde, na capacitação dos professores. Além disso, agir em casos especiais, como em crises convulsivas ou síncopes, bem como auxiliar no cuidado a essas crianças, diminuindo a sobrecarga materna, como nos casos em que a mãe precisa ir até a escola para realizar procedimentos no filho (Milbrath, Soares, Cecagno, Amestoy \& Silveira, 2009).

A presença do enfermeiro na instituição escolar pode ser capaz de minimizar as preocupações decorrentes da doença crônica associada ao ambiente escolar, especialmente referente às preocupações maternas, visto que o profissional pode realizar a assistência à saúde da criança e do adolescente nesse âmbito (Lise, Schwartz, Milbrath \& Nunes, 2019). Dessa forma, tornando-se parte não apenas da manutenção da saúde desse aluno, mas também da prevenção de complicações.

A união entre os serviços de saúde, a escola e a família poderia se tornar facilitadora no enfrentamento da condição crônica na infância, permitindo à criança vivenciar esse processo que irá lhe acompanhar por um longo período ou até mesmo por toda a vida, de uma forma mais tranquila, garantindo a ela o direito de ser criança e de viver plenamente a infância. 


\section{Considerações Finais}

A partir da perspectiva dos profissionais de saúde, o estudo identificou assiduidade, adesão ao tratamento, interesse e atenção dos familiares como fatores que facilitam o cuidado da criança com doença crônica. No entanto, destaca-se que a falta de um profissional de saúde de referência, bem como a ausência de uma rede de apoio, a alta demanda e o número reduzido de profissionais constituem-se em dificuldades enfrentadas pelos profissionais para a efetividade do cuidado à criança com doença crônica e sua família.

Crianças e adolescentes com doenças crônicas têm necessidades singulares em cada fase da doença. Dessa forma, torna-se fundamental o acesso e a disponibilidade de profissionais capacitados para atender essas demandas por meio de um cuidado ampliado, contínuo e resolutivo, capaz de identificar as necessidades biológicas, emocionais e sociais dessa população, efetivando o acolhimento integral.

Este estudo traz contribuições importantes para a prática assistencial dos profissionais de saúde, visto que existe a produção de um cuidado humanizado e integral às famílias de crianças e adolescentes com doença crônica. Sugere-se que sejam desenvolvidos estudos com foco no cuidado multiprofissional, inter e transdisciplinar a essas famílias, com ênfase na criança com doença crônica formando uma rede de cuidados que possa atender às peculiaridades de cada uma, colocando-as como centro de suas ações, por meio de um cuidado humanizado e integral, com ênfase nas políticas públicas, buscando estratégias de cuidado de qualidade e eficácia.

No que se refere às limitações do estudo relaciona-se o fato de terem sido indicados poucos profissionais de saúde como referência, o que pode refletir nos resultados. Acredita-se que a realização de mais estudos com os profissionais de saúde que prestam cuidado a essa população pode favorecer o desenvolvimento de estratégias de cuidado e a ampliação no apoio social às famílias.

\section{Financiamento}

Fundação de Amparo à Pesquisa do Estado do Rio Grande do Sul (FAPERGS/MS/CNPq/SESRS, Edital nº 03/2017 PPSUS - número do auxílio: 00014062)

\section{Referências}

Alves, J. M. N. O., Amendoeira, J. J. P., \& Charepe, Z. B. (2017). A parceria de cuidados pelo olhar dos pais de crianças com necessidades especiais de saúde. Revista Gaúcha de Enfermagem, 38(4),e2016-0070.

Araújo, Y. B. D., Santos, S. R. D., Neves, N. T. D. A. T., Cardoso, É. L. D. S., \& Nascimento, J. A. (2006). Predictive model of hospitalization for children and adolescents with chronic disease. Revista Brasileira de Enfermagem, 73(2), e20180467. https://doi.org/10.1590/0034-7167-2018-0467

Ayres, J., Calazans, G. J., Saletti Filho, H. C., \& França, J. R. I. (2006). Risco, vulnerabilidade e práticas de prevenção e promoção da saúde. In: Campos G, Minayo MCS, Akerman M, Drumond Jr M, Carvalho YM, organizadores. Tratado de Saúde Coletiva. São Paulo: Editora Fiocruz; p. $375-417$.

Barbosa, T. A., Reis, K. M. N., Lomba, G. O., Alves, G. V., \& Braga, P. P. (2016). Supporte network and social support for children with special health care need. Rev Rene, 17(1), 60-66. https://doi.org/10.15253/2175-6783.2016000100009

Benedetto, E. S., \& Silveira, E. (2013). Solo e raízes das dimensões individual, social e programática da vulnerabilidade e as sementes no processo de saúde da criança. Textos contextos, 12(1), 68-84.

Brasil. (2013). Conselho Nacional de Saúde. Resolução n 466, de 12 de dezembro de 2012. Aprova normas regulamentadoras de pesquisas envolvendo seres humanos. Brasília: Diário Oficial da União.

Cabral, I. E., \& Moraes, J. R. M. M. (2015). Family caregivers articulating the social network of a child with special health care needs. Revista Brasileira de Enfermagem, 68 (6),769-76. https://doi.org/10.1590/0034-7167.2015680612i

Cardoso, E. L. S., dos Santos, S. R., de Araújo, Y. B., Neves, N. T. D. A. T., \& do Nascimento, J. A. (2021). Fatores associados à qualidade de vida de cuidadores de crianças e adolescentes com condições crônicas. Revista Gaúcha de Enfermagem, e20190318. https://doi.org/10.1590/19831447.2021.20190318

Clarke, V., \& Braun, V. (2013). Methods: Teaching thematic analysis. The Psychologist, 26(2), 120-123. 
Dantas, K. O., Neves, R. D. F., Ribeiro, K. S. Q. S., Brito, G. E. G. D., \& Batista, M. D. C. (2019). Repercussions on the Family from the birth and care of children with multiple disabilities a qualitative meta-synthesis. Caderno de Saúde Pública, 35 (6), e00157918. http://dx.doi.org/10.1590/0102-311x00157918

Dias, B. C., Marcon, S. S., Reis, P., Lino, I. G. T., Okido, A. C. C., Ichisato, S. M. T., et al. (2020). Family dinamics and social network of families of children with special needs for complex/continuous cares. Revista Gaúcha de Enfermagem, 41:e20190178. http://dx.doi.org/10.1590/1983-1447.2020.20190178

Domenico, C. T., \& Castillo, A. M. C. M. (2017). Social support for the child with type 1 diabetes and their family. Revista de Enfermagem UFPE on line, 11(12), 5020-27. https://doi.org/10.1590/1981-8963-v11i12a15211p5020-5027-2017

Duarte, E. D., Silva, K., Tavares, T. S., Nishimoto, C. L. J., Silva, P. M., \& Sena, R. R. (2015). Care of children with a chronic condition in primary care: challenges to the healthcare model. Texto \& Contexto Enfermagem, 24(4):1009-1017. http://dx.doi.org/10.1590/0104-0707201500003040014

Freitag, V. L., Milbrath, V. M., \& Motta, M. G. C. (2020). Becoming the mother of a child with cerebral palsy: feelings experienced. Psicologia em Estudo, 25:e41608. http://dx.doi.org/10.4024/psicolest.v25i0.41608

Geense, W. W., Van Gaal, B. G. I., Knoll, J. L., Cornelissen, E. A. M., \& Van Achterberg, T. (2017). The support needs of parents having a child with a chronic kidney disease: a focus group study. Child care, health and development, 43(6), 831-838. https://doi.org/10.1111/cch.12476

Leite, D. A. A., de Macedo, L. G., Aliani, M. L., Silva, N. A., Romano, M. C. C., \& Araújo, A. (2020), Origem da obesidade em adolescentes: impressões dos cuidadores familiares assistidos na atenção primária à saúde. Revista de Enfermagem UFPI, 9:e11035. https://doi.org/10.26694/reufpi.v9i0.11035

Lino, I. G. T., Teston, E. F., Marcon, S. S., Andrade, S. M. O. D., Marques, F. R. B., Nass, E. M., \& Marcheti, M. A. (2020). Challenges for the care of families of children with disabilities in primary health care à saúde. Revista Mineira de Enfermagem, 24:e-1340. http://www.dx.doi.org/10.5935/1415.2762.20200077

Lise, F., Schwartz, E., Milbrath, V. M., \& Nunes, N. J. S. (2019). School insertion of children in renal conservative treatment: maternal perceptions. Ciência, cuidado e saúde, 18(1): 1-7. https://doi.org/10.4025/cienccuidsaude.v18i1.45039

Machado, N. A., Nóbrega, V. M., Silva, M. E. A., França, D. B. L., Reichert, A. P. S., \& Collet, N. (2018). Chronic disease in children and adolescentes:profissional-family bond for the promotion of social support. Revista Gaúcha de Enfermagem, 39, e2017-0290. https://doi.org/10.1590/19831447.2018.2017-0290

Machado, N. A., Sousa, M. L. X. F., Silva, M. E. A., Coutinho, S. E. D., Reichert, A. P. S., \& Collet, N. (2015). Dificuldades para a efetivação do acolhimento hospitalar durante a internação de crianças com doença crônica. Revista de Enfermagem UERJ, 23(4):556-561. http://dx.doi.org/10.12957/reuerj.2015.5960

Martins, P. L., Azevedo, C. D. S., \& Afonso, S. B. C. (2018). O papel da família nos planos de tratamento e no cuidado pediátrico hospitalar em condições crônicas complexas de saúde. Saúde e Sociedade, 27(4):1218-29. https://doi.org/10.1590/s0104-12902018170402

Milbrath, V. M., Motta, M. G. C., Gabatz, R. I. B., \& Freitag, V. L. (2017). O nascimento de um filho com paralisia cerebral: um tempo presente inesperado. Revista Interdisciplinar em Cultura e Sociedade,3(n.esp):47-60.

Milbrath, V. M., Soares, D., Cecagno, D., Amestoy, S. C., \& Siqueira, H. C. (2009). Inserção social da criança portadora de necessidades especiais: a perspectiva materna. Cogitare enfermagem, 14(2):311-17. http://dx.doi.org/10.5380/ce.v14i2.15623

Minayo, M. C. S. Técnicas de análise do material qualitativo. In: Minayo, M. C. S. O desafio do conhecimento: pesquisa qualitativa em saúde. (2013). 13ed. São Paulo (Hucitec).

Nóbrega, V. M., Damasceno, S. S., Rodrigues, P. F., Reichert, A. P. S., \& Collet, N. (2013). Atenção à criança com doença crônica na estratégia saúde da família. Cogitare enfermagem, 18(1):57-63. http://dx.doi.org/10.5380/ce.v18i1.28517

Nóbrega, V. M., Silva, M. E. A., Fernandes, L. T. B., Viera, C. S., Reichert, A. P. S., \& Collet, N. (2017). Chronic disease in childhood and adolescence: continuity of care in the health care network. Revista Escola de Enfermagem USP, 51:e03226. https://doi.org/10.1590/s1980-220x2016042503226

Pais, S. C., \& Menezes, I. (2019). How do we live with chronic disease? A rights-based approach promoting the wellbeing od children with chronic disease. Ciência e Saúde Coletiva, 24(10): 3663-72. https://doi.org/10.1590/1413-812320182410.17932019

Pedrosa, R. K. B., Guedes, A. T. A., Soares, A. R., Vaz, E. M. C., Collet, N., \& Reichert, A. P. D. S. (2020). Itinerary of children with microcephaly in the health care network. Escola Anna Nery Revista de Enfermagem, 24(3): e20190263. https://doi.org/10.1590/2177-9465-ean-2019-0263

Ramos, R. M., Nóbrega, V. M., Fernandes, L. T. B., Machado, A. N., \& Collet, N. (2017). Parental care to children and adolescente with chronic disease: maternal perception. Revista Gaúcha de Enfermagem, 38(3):e2016-0006. https://doi.org/10.1590/1983-1447.2017.03.2016-0006

Salvador, M. S., Gomes, G. C., Oliveira, P. K., Gomes, V. L. O., Busanello, J., \& Xavier, D. M. (2015). Strategies of families in the care of children with chronic diseases. Texto \& Contexto Enfermagem, 24(3): 662-9. http://dx.doi/10.1590/0104-07072015000300014

Silva, M. E. A., Reichert, A. P. S., Souza, F. A. Z., Pimenta, E. A. G., \& Collet, N. (2018). Chronic disease in childhood and adolescence: family bondsin the healthcare network. Texto \& Contexto Enfermagem, 27(2):1-11. https://doi.org/10.1590/0104-070720180004460016

Silva, M. E. D. A., Moura, F. M. D., Albuquerque, T. M., Reichert, A. P. D. S., \& Collet, N. (2017). Network and social support in children with chronic disease: understanding the children's perception. Texto \& Contexto-Enfermagem, 26(1):e6980015. http://dx.doi.org/10.1590/0104-07072017006980015

Souza, M. H. N., Nóbrega, V. M., \& Collet, N. (2020). Social network of children with cronic disease: knowledge and practice of nursing. Revista Brasileira de Enfermagem, 73(2):e20180371. https://doi.org/10.1590/0034-7167-2018-0371

Souza, R. L. A., Mutti, C. F., Santos, R. P., Oliveira, D. C., Okido, A. C. C., Jantsch, L. B., et al. (2021). A hospitalização na percepção de crianças e adolescentes em tratamento oncológico. Revista Gaúcha de Enfermagem, 42:e20200122. https://doi.org/10.1590/1983-1447.2021.20200122

Vaz, J. C. (2018). Vulnerabilidade vivenciada pelos familiares/cuidadores de crianças com condição crônica. Dissertação (Mestrado). Programa de Pós Graduação em Ciências da Saúde da Universidade Federal de Pelotas. 209p. 